\title{
DYNAMIC RESPONSE OF A SPUR GEAR SYSTEM WITH UNCERTAIN PARAMETERS
}

\author{
Ahmed Guerine \\ Laboratory Optimization and Reliability in Structural Mechanics LOFIMS, Mechanical Engineering Department, \\ National Institute of Applied Sciences of Rouen, Cedex, France and \\ Mechanics, Modelling and Manufacturing Laboratory LA2MP, Mechanical Engineering Department, \\ National School of Engineers of Sfax, Sfax, Tunisia; e-mail: ahmedguerine@gmail.com
}

ABDelkhalak El Hami

Laboratory Optimization and Reliability in Structural Mechanics LOFIMS, Mechanical Engineering Department, National Institute of Applied Sciences of Rouen, Cedex, France

Lassaad Walha, Tahar Fakhfakh, Mohamed Haddar

Mechanics, Modelling and Manufacturing Laboratory LA2MP, Mechanical Engineering Department, National School of Engineers of Sfax, Sfax, Tunisia

\begin{abstract}
In this paper, we propose a new approach for taking into account uncertainties based on the projection on polynomial chaos. The new approach is used to determine the dynamic response of a spur gear system with uncertainty associated to gear system parameters. The simulation results are obtained by the polynomial chaos approach for dynamic analysis under uncertainty. The proposed approach is an efficient probabilistic tool for uncertainty propagation. It has been found to be an interesting alternative to the parametric studies. The polynomial chaos results are compared with Monte Carlo simulations.
\end{abstract}

Keywords: uncertainty, spur gear system, polynomial chaos approach, gear parameters

\section{Introduction}

The gearing is the best solution to transmit rotational motion and torque, which offers numerous advantages (Dalpiaz et al., 1996) including mechanical reliability. Furthermore, its mechanical efficiency is of the order of 0.96 to 0.99 . But today, several applications require gear transmissions to be more and more reliable, light and having long service life. This requires control of the acoustic broadcast and vibratory behavior of such gearings (Begg et al., 2000).

Several parametric studies (coefficient of friction, assembly of defects, manufacturing defects, eccentricity default,...) have shown great sensitivity of the dynamic behavior of gear systems. However, these parameters assume strong dispersions (Guerine et al. 2015a,b). Therefore, it becomes necessary to take into account the uncertainties to ensure the robustness of the analysis. Also, there are several studies on reliability in vibration structures taking into account these uncertainties (Abo Al-kheer et al., 2011; El Hami et al., 1993, 2009; El Hami and Radi, 1996).

Several methods are proposed in the literature. Monte Carlo (MC) simulation is a well-known technique in this field (Fishman, 1996). It can give the entire probability density function of any system variable, but it is often too costly since a great number of samples are required for reasonable accuracy. Parallel simulation (Papadrakakis and Papadopoulos, 1999) and proper orthogonal decomposition (Lindsley and Beran, 2005) are some solutions proposed to circumvent the computational difficulties of the MC method.

Polynomial chaos expansion (PCE) is presented in the literature as a more efficient probabilistic tool for uncertainty propagation. It was first introduced by Wiener (1938) and pioneered 
by Ghanem and Spanos (1991) who used Hermite orthogonal polynomials to model stochastic processes with Gaussian random variables. The exponential convergence of such an expansion was shown by Cameron and Martin (1947) and then was generalized to various continuous and discrete distributions using orthogonal polynomials from the so called Askey-scheme (Askey and Wilson, 1985; Xiu and Karniadakis, 2003).

Polynomial chaos (PC) gives a mathematical framework to separate stochastic components of a system response from deterministic ones. The stochastic Galerkin method (Babuska et al., 2004; Le Maître et al., 2001), collocation and regression methods (Isukapalli et al., 1998a,b; Crestaux et al. 2009) are used to compute deterministic components called stochastic modes in an intrusive and non-intrusive manner while random components are concentrated in the polynomial basis used. Non-intrusive procedures prove to be more advantageous for stochastic dynamic systems since they need no modifications of the system model, contrary to the intrusive method. In the latter, Galerkin techniques are used to generate a set of deterministic coupled equations from a stochastic system model, and then a suitable algorithm is adapted to obtain stochastic modes.

The capabilities of polynomial chaos have been tested in numerous applications, such as treating uncertainties in environmental and biological problems (Isukapalli et al., 1998a,b), in multibody dynamic systems (Sandu et al., 2006a,b), in solving ordinary and partial differential equations (Williams, 2006; Xiu and Karniadakis, 2002), in component mode synthesis techniques (El Hami and Radi, 1996, Sarsri et al., 2011) and parameter estimation (Saadet al., 2007; Blanchard et al., 2009; Smith et al., 2007).

The main originality of the present paper is that the uncertainty of gear system parameters in the dynamic behavior study of the one stage gear system is taken into account. The main objective is to investigate the capabilities of the new method to determine the dynamic response of a spur gear system subject to uncertain gear parameters. So, an eight degree of freedom system modelling the dynamic behavior of a spur gear system is considered. The modelling of a one-stage spur gear system is presented in Section 2. In the next Section, the theoretical basis of the polynomial chaos is presented. In Section 4, the equations of motion for the eight degrees of freedom are presented. Numerical results are presented in Section 5. Finally in Section 6, to conclude, some comments are made based on the study carried out in this paper.

\section{Modelling of a one-stage gear system}

The global dynamic model of a one-stage gear system is shown in Fig. 1. This model is composed of two blocks $(j=1,2)$. Each block $(j)$ is supported by a flexible bearing whose bending stiffness is $k_{j}^{x}$ and the traction-compression stiffness is $k_{j}^{y}$.

Wheels (11) and (22) characterize respectively the motor side and the receiving side. The shafts $(j)$ admit some torsional stiffness $k_{j}^{\theta}$.

Angular displacements of each wheel are noticed by $\theta_{(i, j)}$ with the indices $j=1$ to 2 designating the number of the block, and $i=1$ to 2 designate the two wheels of each block. Moreover, linear displacements of the bearing denoted by $x_{j}$ and $y_{j}$ are measured in the plane which is orthogonal to the wheels axis of rotation.

In this study, we model the gear mesh stiffness variation $k(t)$ by a square wave form (Fig. 2). The gear mesh stiffness variation can be decomposed in two elements: an average component denoted by $k c$, and a time variant one noted by $k v(t)$ (Walha et al., 2009).

The extreme values of the mesh stiffness are defined by

$$
k_{\min }=-\frac{k c}{2 \varepsilon^{\alpha}} \quad k_{\max }=-k_{\min } \frac{2-\varepsilon^{\alpha}}{\varepsilon^{\alpha}-1}
$$




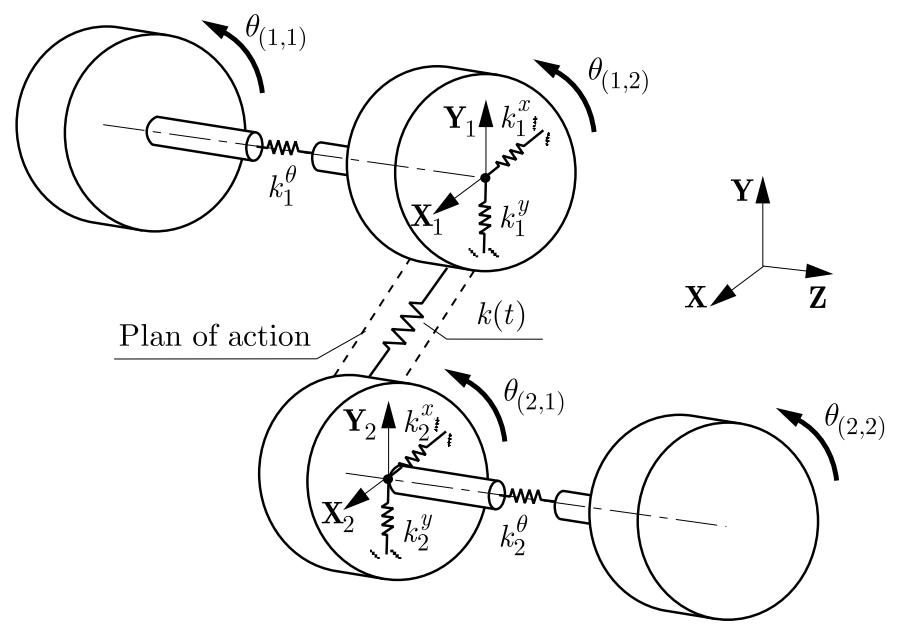

Fig. 1. Global dynamic model of the one stage gear system

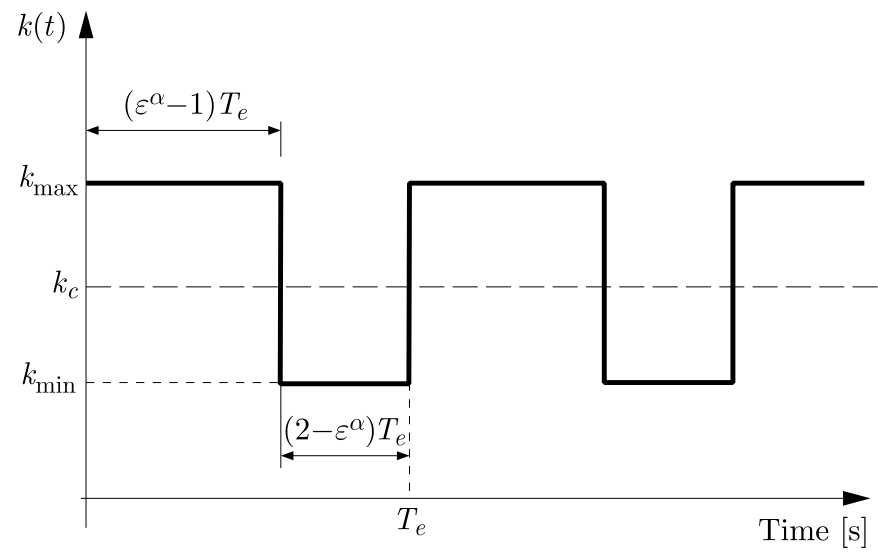

Fig. 2. Modelling of the mesh stiffness variation

where $\varepsilon^{\alpha}$ and $T e$ represent respectively the contact ratio and mesh period corresponding to the two gearmesh contacts.

\section{Polynomial chaos approach}

In this Section, we propose a new method based on the projection on polynomial chaos. This method consists in projecting stochastic desired solutions on a basis of orthogonal polynomials in which variables are Gaussian orthonormal. The properties of the base polynomial are used to generate a linear system of equations by means of projection. The resolution of this system leads to an expansion of the solution on the polynomial basis, which can be used to calculate the moments of the random solution. With this method, we can easily calculate the dynamic response of a mechanical system.

Let us consider a multi-degrees of freedom linear system with mass and stiffness matrices $\mathbf{M}_{T}$ and $\mathbf{K}_{T}$, respectively. The equations of motion describing forced vibration of a linear system are

$$
\mathbf{M}_{T} \ddot{\mathbf{u}}_{T}(t)+\mathbf{K}_{T} \mathbf{u}_{T}(t)=\mathbf{f}_{T}(t)
$$

where $\mathbf{u}_{T}$ is the nodal displacement vector and $\mathbf{f}_{T}$ is the external excitation. 
The chaotic polynomials $\psi_{m}$ corresponding to the multidimensional Hermite polynomials obtained by formula (3.2)

$$
\psi_{m}\left(\alpha_{1}, \ldots, \alpha_{P}\right)=(-1)^{P} \mathrm{e}^{\left(\frac{1}{2} \boldsymbol{\alpha}^{\mathrm{T}} \boldsymbol{\alpha}\right)} \frac{\partial^{P} \mathrm{e}^{\left(\frac{1}{2} \boldsymbol{\alpha}^{\mathrm{T}} \boldsymbol{\alpha}\right)}}{\partial \alpha_{1} \cdots \partial \alpha_{P}}
$$

where $\boldsymbol{\alpha}$ is the vector grouping the random variables

$$
\boldsymbol{\alpha}^{\mathrm{T}}=\left[\alpha_{1}, \ldots, \alpha_{P}\right]
$$

The random mass and stiffness matrices $\mathbf{M}_{T}$ and $\mathbf{K}_{T}$ of the mechanical system can be written as

$$
\mathbf{M}_{T}=\left[\mathbf{M}_{T}\right]_{0}+\widetilde{\mathbf{M}}_{T} \quad \mathbf{K}_{T}=\left[\mathbf{K}_{T}\right]_{0}+\widetilde{\mathbf{K}}_{T}
$$

where $\left[\mathbf{M}_{T}\right]_{0}$ and $\left[\mathbf{K}_{T}\right]_{0}$ are deterministic matrices, $\widetilde{\mathbf{M}}_{T}$ and $\widetilde{\mathbf{K}}_{T}$ correspond to the random part of the mass and stiffness matrices. $\widetilde{\mathbf{M}}_{T}$ and $\widetilde{\mathbf{K}}_{T}$ are rewritten from an expression of the Karhunen-Loeve type (Ghanem and Spanos, 1991) in the following form

$$
\widetilde{\mathbf{M}}_{T}=\sum_{p=1}^{P}\left[\mathbf{M}_{T}\right]_{p} \alpha_{p} \quad \widetilde{\mathbf{K}}_{T}=\sum_{p=1}^{P}\left[\mathbf{K}_{T}\right]_{p} \alpha_{p}
$$

where $\alpha_{p}$ is an independent Gaussian random variable which may correspond to the first polynomial $\psi_{p}$, while the matrices $\left[\mathbf{M}_{T}\right]_{p}$ and $\left[\mathbf{K}_{T}\right]_{p}$ are deterministic.

We pose $\alpha_{0}=1$, we can write then

$$
\mathbf{M}_{T}=\sum_{p=0}^{P}\left[\mathbf{M}_{T}\right]_{p} \alpha_{p} \quad \mathbf{K}_{T}=\sum_{p=0}^{P}\left[\mathbf{K}_{T}\right]_{p} \alpha_{p}
$$

In the same way, we can write for $\mathbf{f}_{T}$

$$
\mathbf{f}_{T}=\sum_{p=0}^{P}\left\{\mathbf{f}_{T}\right\}_{p} \alpha_{p}
$$

The dynamic response is obtained by solving the following equation, knowing that the initial conditions are predefined

$$
\mathbf{K}_{e q} \mathbf{u}_{T}(t+\Delta t)=\mathbf{F}_{e q}
$$

where

$$
\begin{aligned}
& \mathbf{K}_{e q}=\mathbf{K}_{T}+a_{0} \mathbf{M}_{T} \\
& \mathbf{F}_{e q}=\mathbf{f}_{T}(t+\Delta t)+\mathbf{M}_{T}\left[a_{0} \mathbf{u}_{T}(t)+a_{1} \dot{\mathbf{u}}_{T}(t)+a_{2} \ddot{\mathbf{u}}_{T}(t)\right]
\end{aligned}
$$

where

$$
a_{0}=\frac{1}{A \Delta t^{2}} \quad a_{1}=\frac{B}{A \Delta t} \quad a_{2}=\frac{1}{A \Delta t}
$$

$A$ and $B$ are the parameters of the Newmark method. $\mathbf{u}_{T}(t+\Delta t)$ is decomposed on polynomials to $P$ Gaussian random orthnormal variables, where $P$ is the number of random variables

$$
\mathbf{u}_{T}(t+\Delta t)=\sum_{n=0}^{N}\left\{\mathbf{u}_{T}(t+\Delta t)\right\}_{n} \psi_{n}\left(\left\{\boldsymbol{\alpha}_{i}\right\}_{i=1}^{P}\right)
$$


where $N$ is the polynomial chaos order. It is the degree of polynomial chaos. $\mathbf{K}_{e q}$ and $\mathbf{F}_{e q}$ are written in the following form

$$
\begin{aligned}
\mathbf{K}_{e q} & =\sum_{p=0}^{P}\left[\mathbf{K}_{T}\right]_{p} \alpha_{p}+a_{0} \sum_{p=0}^{P}\left[\mathbf{M}_{T}\right]_{p} \alpha_{p}=\sum_{p=0}^{P}\left[\mathbf{K}_{e q 2}\right]_{p} \alpha_{p} \\
\mathbf{F}_{e q} & =\sum_{p=0}^{P}\left\{\mathbf{f}_{T}(t+\Delta t)\right\}_{p} \alpha_{p}+\sum_{p=0}^{P}\left[\mathbf{M}_{T}\right]_{p} \alpha_{p}\left(a_{0}\left\{\mathbf{u}_{T}(t)\right\}_{0}+a_{1}\left\{\dot{\mathbf{u}}_{T}(t)\right\}_{0}+a_{2}\left\{\ddot{\mathbf{u}}_{T}(t)\right\}_{0}\right) \\
& =\sum_{p=0}^{P}\left\{\mathbf{F}_{e q 2}\right\}_{p} \alpha_{p}
\end{aligned}
$$

Substituting Eqs. (3.11) and (3.12) into Eq. (3.8) and forcing the residual to be orthogonal to the space spanned by the polynomial chaos $\psi_{m}$, yields the following system of linear equations

$$
\sum_{p=0}^{P} \sum_{n=0}^{N}\left[\mathbf{K}_{e q 2}\right]_{p}\left\{\mathbf{u}_{T}\right\}_{n}\left\langle\alpha_{p} \psi_{n} \psi_{m}\right\rangle=\sum_{p=0}^{P}\left\{\mathbf{F}_{e q 2}\right\}_{p}\left\langle\alpha_{p} \psi_{m}\right\rangle \quad m=0,1, \ldots, N
$$

where $\langle\cdot \cdot\rangle$ denotes the inner product defined by the expectation operator.

This algebraic equation can be rewritten in a more compact matrix form as

$$
\left[\begin{array}{ccccc}
\mathbf{D}^{00} & & \cdots & & \mathbf{D}^{0 N} \\
& \ddots & & & \\
\vdots & & \mathbf{D}^{i j} & & \vdots \\
& & & \ddots & \\
\mathbf{D}^{N 0} & & \cdots & & \mathbf{D}^{N N}
\end{array}\right]\left\{\begin{array}{c}
\left\{\mathbf{u}_{T}(t+\Delta t)\right\}_{0} \\
\vdots \\
\left\{\mathbf{u}_{T}(t+\Delta t)\right\}_{j} \\
\vdots \\
\left\{\mathbf{u}_{T}(t+\Delta t)\right\}_{N}
\end{array}\right\}=\left\{\begin{array}{c}
\left\{\mathbf{f}^{(0)}\right. \\
\vdots \\
\left\{\mathbf{f}^{(j)}\right. \\
\vdots \\
\left\{\mathbf{f}^{(N)}\right.
\end{array}\right\}
$$

where

$$
\mathbf{D}^{(i j)}=\sum_{p=0}^{P}\left[\mathbf{K}_{e q 2}\right]_{p}\left\langle\alpha_{p} \psi_{i} \psi_{j}\right\rangle \quad \mathbf{f}^{(j)}=\sum_{p=0}^{P}\left\{\mathbf{F}_{e q 2}\right\}_{p}\left\langle\alpha_{p} \psi_{j}\right\rangle
$$

Due to the orthogonality of the polynomials, most terms $\left\langle\alpha_{p} \psi_{n} \psi_{m}\right\rangle$ are zero. Indeed, we have

$$
\left\langle\psi_{i} \psi_{j}\right\rangle_{i \neq j}=0
$$

After resolution of algebraic system (3.14), the mean values and the variances of the dynamic response are given by the following relationships

$$
E\left[\mathbf{u}_{T}\right]=\left\{\mathbf{u}_{T}(t+\Delta t)\right\}_{0} \quad \operatorname{Var}\left[\mathbf{u}_{T}\right]=\sum_{n=1}^{N}\left(\left\{\mathbf{u}_{T}(t+\Delta t)\right\}_{n}\right)^{2} \psi_{j}^{2}
$$




\section{Equations of motion}

The equations of motion describing the dynamic behavior of our system (Fig. 1) are obtained by applying Lagrange formulation, and are given by

$$
\begin{aligned}
& m \ddot{x}_{1}+k_{1}^{x} x_{1}+\sin (\alpha) k(t)\left\langle L^{\delta}\right\rangle \mathbf{Q}=0 \\
& m \ddot{y}_{1}+k_{1}^{y} y_{1}+\cos (\alpha) k(t)\left\langle L^{\delta}\right\rangle \mathbf{Q}=0 \\
& m \ddot{x}_{2}+k_{2}^{x} x_{2}-\sin (\alpha) k(t)\left\langle L^{\delta}\right\rangle \mathbf{Q}=0 \\
& m \ddot{y}_{2}+k_{2}^{y} y_{2}-\cos (\alpha) k(t)\left\langle L^{\delta}\right\rangle \mathbf{Q}=0 \\
& I \ddot{\theta}_{(1,1)}+k_{1}^{\theta}\left(\theta_{(1,1)}-\theta_{(1,2)}\right)=C m \\
& I \ddot{\theta}_{(1,2)}-k_{1}^{\theta}\left(\theta_{(1,1)}-\theta_{(1,2)}\right)+r_{(1,2)}^{b} k(t)\left\langle L^{\delta}\right\rangle \mathbf{Q}=0 \\
& I \ddot{\theta}_{(2,1)}-k_{2}^{\theta}\left(\theta_{(2,1)}-\theta_{(2,2)}\right)-r_{(2,1)}^{b} k(t)\left\langle L^{\delta}\right\rangle \mathbf{Q}=0 \\
& I \ddot{\theta}_{(2,2)}+k_{2}^{\theta}\left(\theta_{(2,1)}-\theta_{(2,2)}\right)=0
\end{aligned}
$$

where $\left\langle L^{\delta}\right\rangle$ is defined by

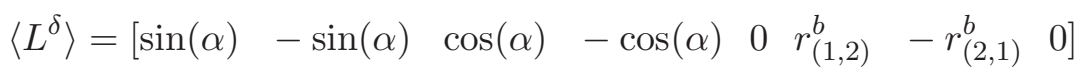

(4.9) $r_{(1,2)}^{b}, r_{(2,1)}^{b}$ represent the base radius of gears, $\alpha$ is the pressure angle. $\mathbf{Q}(t)$ is the vector of generalized coordinates

$$
\mathbf{Q}(t)=\left[\begin{array}{llllllll}
x_{1} & y_{1} & x_{2} & y_{2} & \theta_{(1,1)} & \theta_{(1,2)} & \theta_{(2,1)} & \theta_{(2,2)}
\end{array}\right]^{\mathrm{T}}
$$

\section{Numerical simulation}

The technological and dimensional features of the one-stage gear system are summarized in Table 1.

Table 1. System parameters

\begin{tabular}{|l|l|}
\hline Material: 42CrMo4 & $\rho=7860 \mathrm{Kg} / \mathrm{m}^{3}$ \\
\hline Motor torque & $C m=200 \mathrm{~N} \mathrm{~m}$ \\
\hline Bearing stiffness & $k_{j}^{x}=10^{7} \mathrm{~N} / \mathrm{m}, k_{j}^{y}=10^{7} \mathrm{~N} / \mathrm{m}$ \\
\hline Torsional stiffness of shaft & $k_{j}^{\theta}=10^{5} \mathrm{~N} \mathrm{~m} / \mathrm{rad}$ \\
\hline Number of teeth & $Z(12)=40, Z(21)=50$ \\
\hline Module of teeth & module $=4.10^{-3} \mathrm{~m}$ \\
\hline Contact ratio & $\varepsilon^{\alpha}=1.7341$ \\
\hline The pressure angle & $\alpha=20^{\circ}$ \\
\hline
\end{tabular}

In this Section, numerical results are presented for the new formulations derived in Section 3. The polynomial chaos results are compared with Monte Carlo findings for 100000 simulations.

The mass $m$ and the moment of inertia $I$ of the gears are supposed to be random variables and defined as follows

$$
m=m_{0}+\sigma_{m} \xi \quad I=I_{0}+\sigma_{I} \xi
$$

where $\xi$ is the zero mean value Gaussian random variable, $m_{0}$ and $I_{0}$ are the mean values and $\sigma_{m}$ and $\sigma_{I}$ are the associated standard deviations.

The mean value and standard deviation of the dynamic component of the linear displacement of the first bearing in the two directions $x$ and $y$ have been calculated by the polynomial chaos 
approach. The obtained results are compared with those given from Monte Carlo simulations for 100000 operations. The results are plotted in Figs. 3a, 4a, 5a and 6a for $\sigma_{m}=\sigma_{I}=2 \%$ and in Figs. 3b, 4b, 5b and 6b for $\sigma_{m}=\sigma_{I}=5 \%$.

These figures show that the obtained solutions oscillate around the Monte Carlo simulations which are the reference solution. It can be seen that for a small standard deviation $\sigma_{m}=\sigma_{I}=2 \%$, the polynomial chaos solutions in the second order polynomial provide a very good accuracy as compared with the Monte Carlo results. When the standard deviation increases, the error increases.
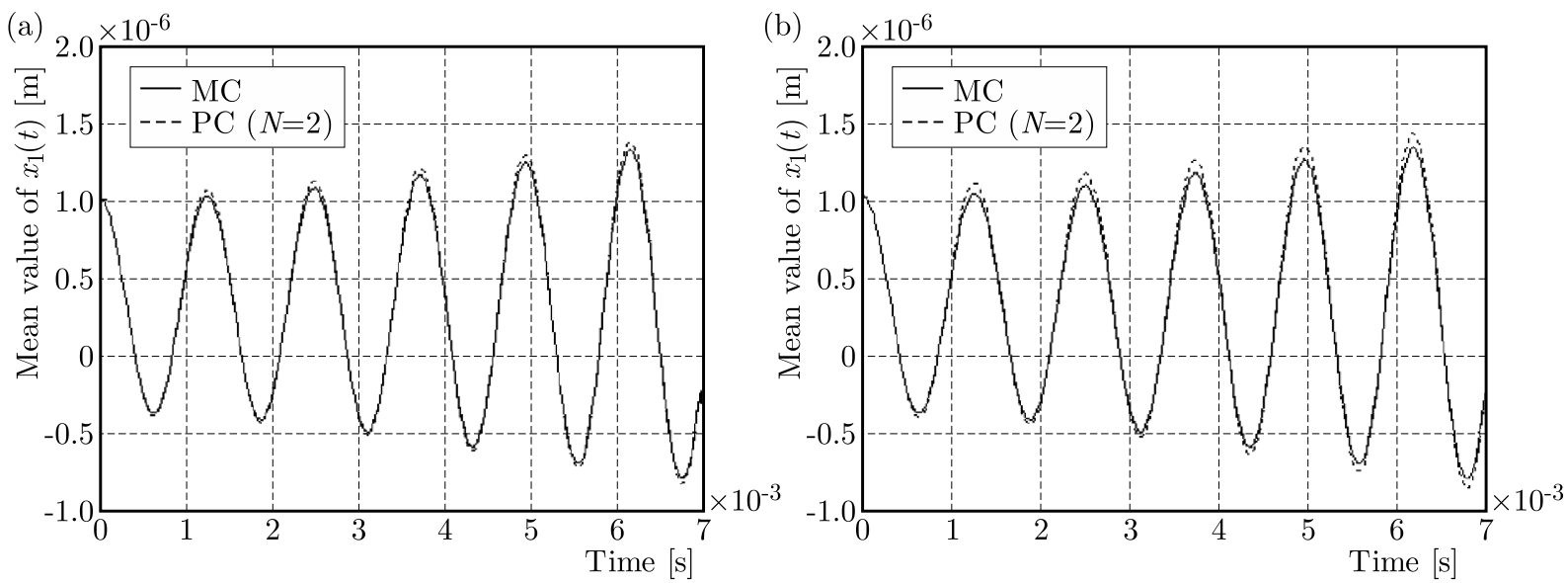

Fig. 3. Mean value of $x_{1}(t)$ for (a) $\sigma_{m}=\sigma_{I}=2 \%$ and (b) $\sigma_{m}=\sigma_{I}=5 \%$
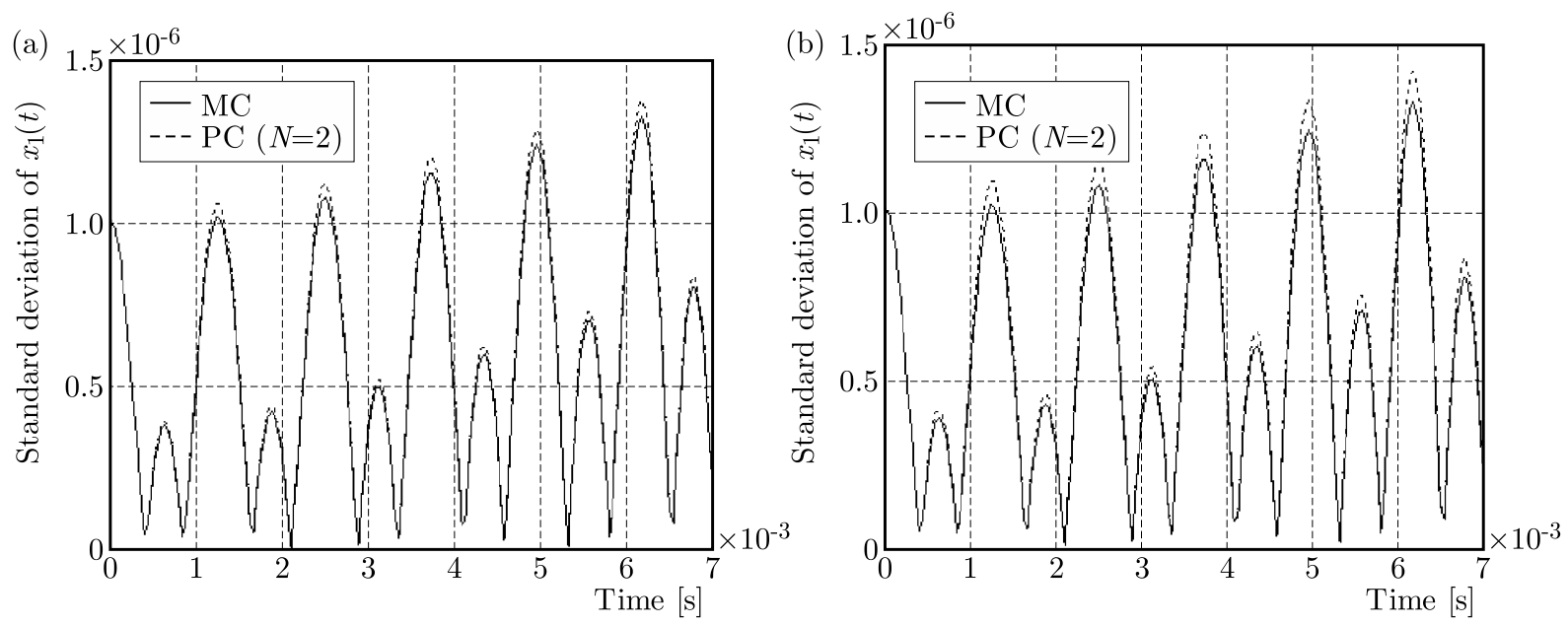

Fig. 4. Standard deviation of $x_{1}(t)$ for (a) $\sigma_{m}=\sigma_{I}=2 \%$ and (b) $\sigma_{m}=\sigma_{I}=5 \%$

The mean values of the dynamic component of the angular displacements $\theta_{(1,1)}$ and $\theta_{(2,1)}$ are presented in Figs. 7a and $7 \mathrm{~b}$ for $\sigma_{m}=\sigma_{I}=5 \%$. The polynomial chaos results are compared with the Monte Carlo simulations for 100000 operations. The dynamic response of the angular displacement as predicted by polynomial chaos calculations matches exactly with that of the Monte Carlo analysis. $N=8$ has been used for the PC model, and it is seen to be enough to capture the dynamic response of the angular displacement of our system.

The mean value and standard deviation of the dynamic component of the linear displacement of the second bearing in the two directions $x$ and $y$ are presented in Figs. 8 and 9 for $\sigma_{m}=\sigma_{I}=10 \%$. 

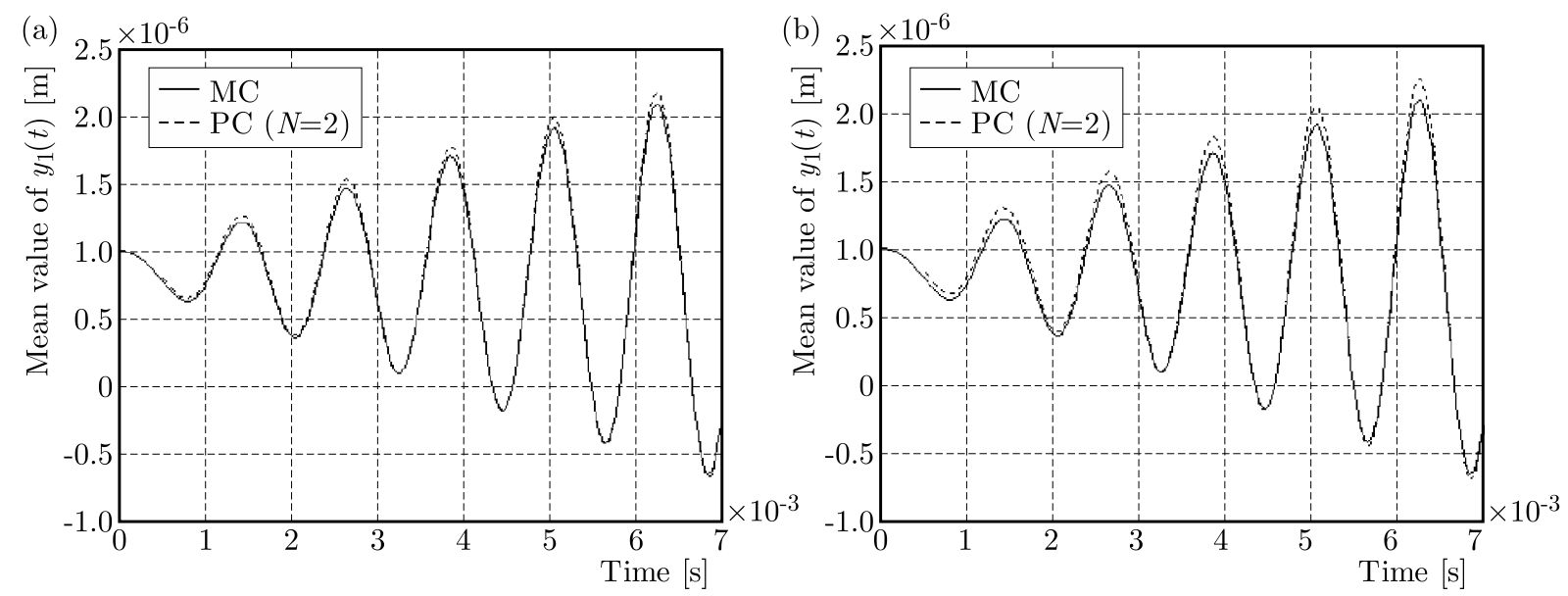

Fig. 5. Mean value of $y_{1}(t)$ for (a) $\sigma_{m}=\sigma_{I}=2 \%$ and (b) $\sigma_{m}=\sigma_{I}=5 \%$
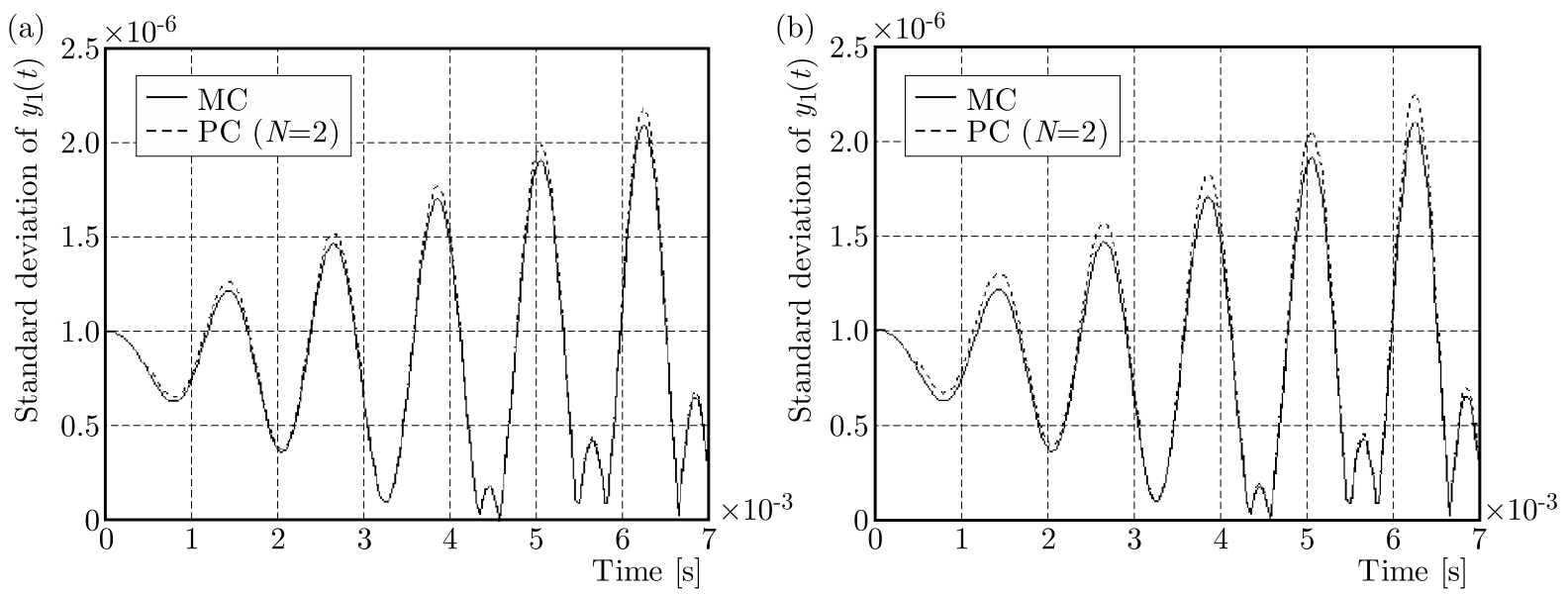

Fig. 6. Standard deviation of $y_{1}(t)$ for (a) $\sigma_{m}=\sigma_{I}=2 \%$ and (b) $\sigma_{m}=\sigma_{I}=5 \%$
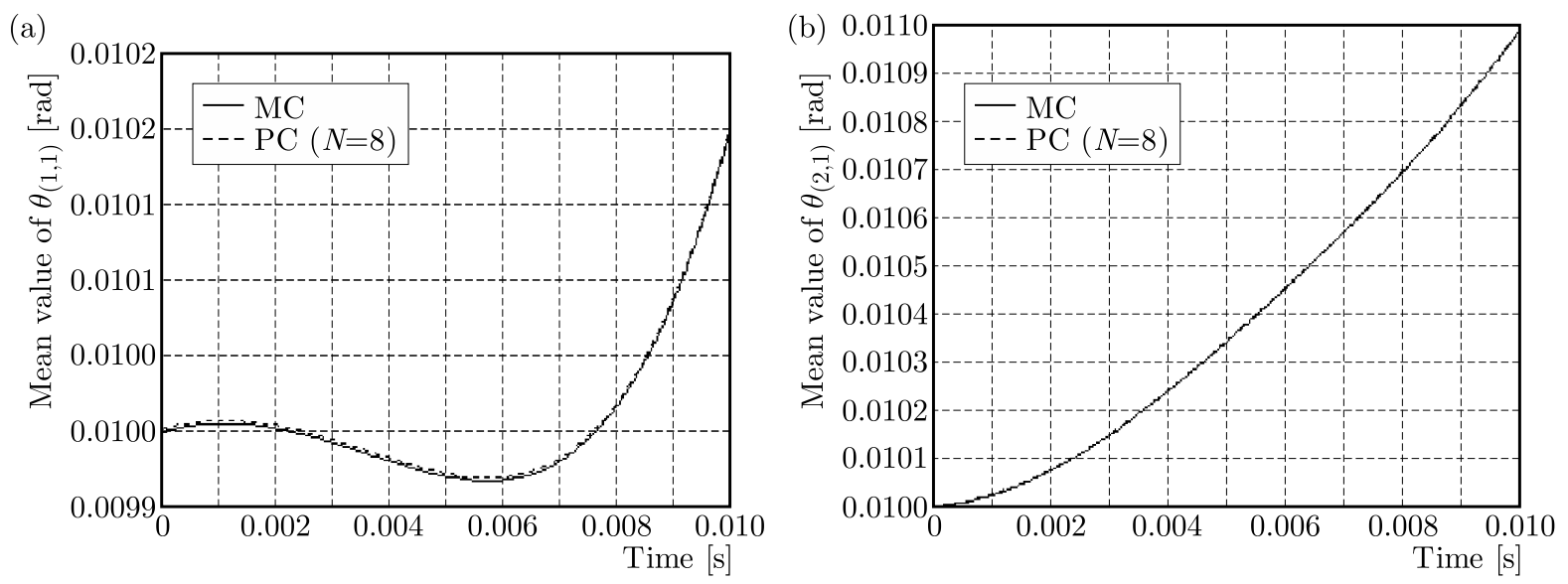

Fig. 7. Mean value of (a) $\theta_{(1,1)}(t)$ and (b) $\theta_{(2,1)}(t)$ for $\sigma_{m}=\sigma_{I}=5 \%$

The polynomial chaos results are compared with the Monte Carlo results for 100000 simulations. It is evident from these figures that the case $N=2$ clearly has not enough chaos terms to represent the output. As $N$ increases, the results seem to become better, and for $N=8$, the dynamic response of the linear displacement of the second bearing with polynomial chaos values almost exactly match with the Monte Carlo simulation results. 

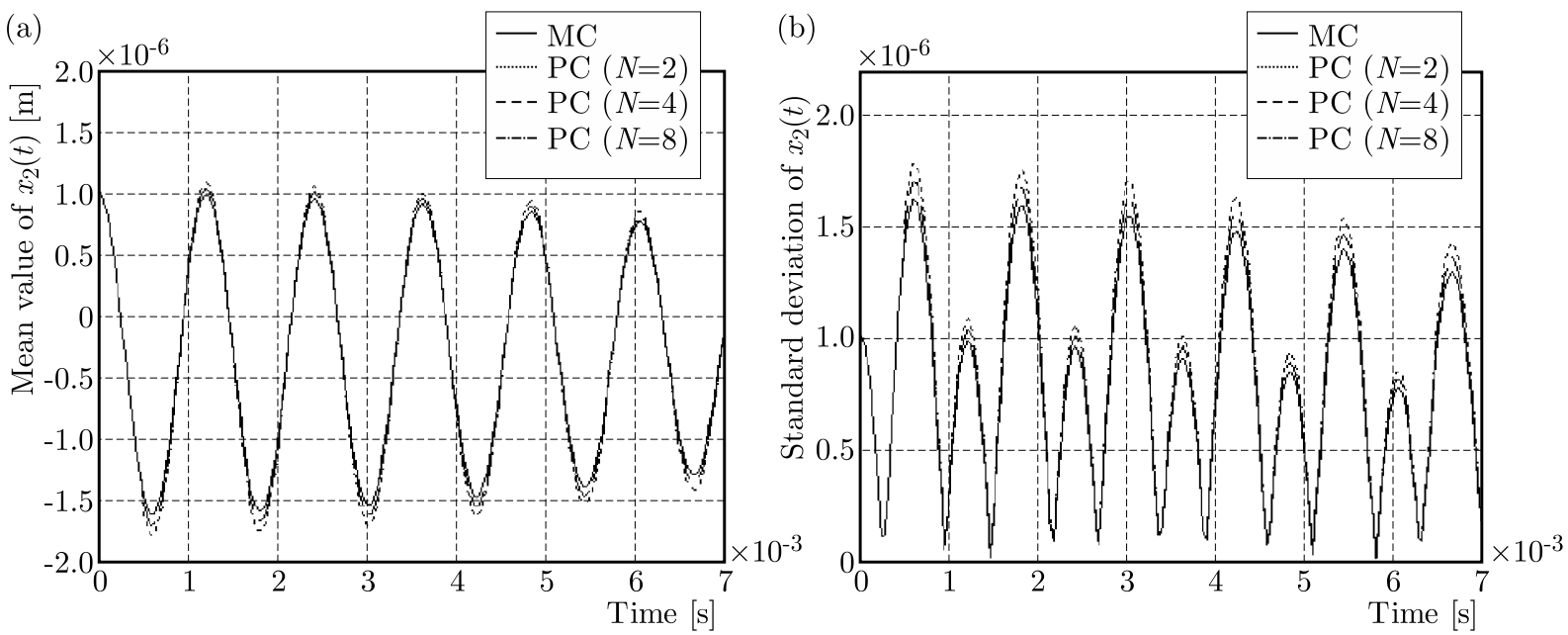

Fig. 8. (a) Mean value of $x_{2}(t)$ for $\sigma_{m}=\sigma_{I}=10 \%$, (b) standard deviation of $x_{2}(t)$ for $\sigma_{m}=\sigma_{I}=10 \%$
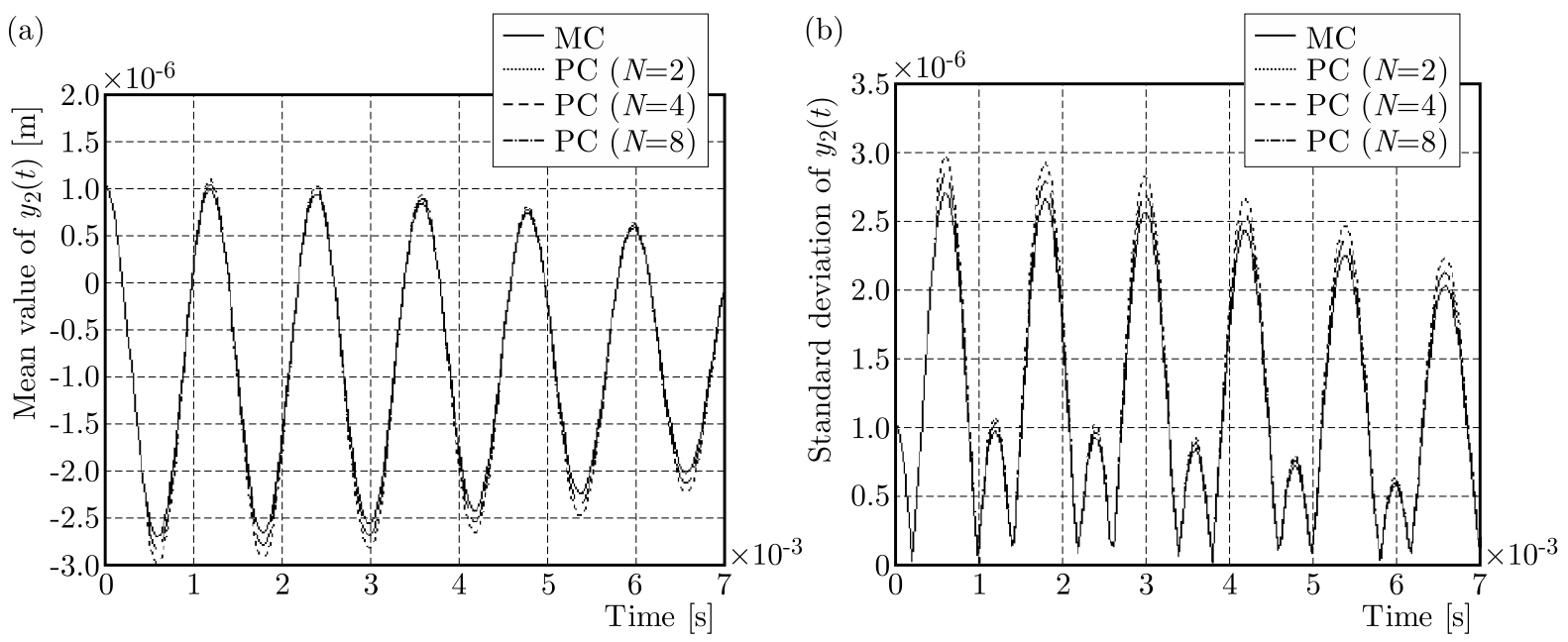

Fig. 9. (a) Mean value of $y_{2}(t)$ for $\sigma_{m}=\sigma_{I}=10 \%$, (b) standard deviation of $y_{2}(t)$ for $\sigma_{m}=\sigma_{I}=10 \%$

\section{Conclusion}

This paper has presents an approach to a one-stage gear system based on the polynomial chaos method while explicitly considering the uncertainty in parameters of the gear. The main results of the present study show that the polynomial chaos method may be an efficient tool to take into account the dispersions of the gear parameters. An interesting perspective is to apply this method to a system with higher degrees of freedom like an epicyclic gear system. Further work in this context is in progress.

\section{References}

1. Abo Al-kheer A., El-Hami A., Kharmanda M.G., Mouazen A.M., 2011, Reliability-based design for soil tillage machines, Journal of Terramechanics, 48, 1, 57-64

2. Askey R., Wilson J., 1985, Some basic hypergeometric polynomials that generalize jacobi polynomials, Memoirs of the American Mathematical Society, 319

3. Babuska I., Nobile F., Tempone R., 2007, A stochastic collocation method for elliptic partial differential equations with random input data, SIAM Journal on Numerical Analysis, 45, 1005-1034 
4. Babuska I., Tempone R., Zouraris G.E., 2004, Galerkin finite element approximation of stochastic elliptic partial differential equations, SIAM Journal on Scientific Computing, 24, 619-644

5. Begg C.D., Byington C.S., Maynard K., 2000, Dynamic simulation of mechanical fault transition, Proceedings of the 54th Meeting of the Society for Machinery Failure Prevention Technology, Virginia Beach

6. Blanchard E., Sandu A., Sandu C., 2009, Parameter estimation for mechanical systems via an explicit representation of uncertainty, Engineering Computations, 26, 5, 541-569

7. Cameron H., Martin W., 1947, The orthogonal development of nonlinear functional in series of Fourier-Hermite functional, Annals of Mathematics, 48, 385-392

8. Crestaux T., Le Maitre O., Martinez J.M., 2009, Polynomial chaos expansion for sensitivity analysis, Reliability Engineering and System Safety, 94, 1161-1172

9. Dalpiaz G., Rivola A., Rubini R., 1996, Dynamic modeling of gear systems for condition monitoring and diagnostics, Congress on Technical Diagnostics

10. El Hami A., Lallement G., Minottiand P., Cogan S., 1993, Methods that combine finite group theory with component mode synthesis in the analysis of repetitive structures, International Journal Computers and Structures, 48, 975-982

11. El Hami A., Radi B., 1996, Some decomposition methods in the analysis of repetitive structures, International Journal Computers and Structures, 58, 5, 973-980

12. El Hami A., Radi A., Cherouat A., 2009, The frictional contact of the shaping of the composite fabric, International Journal of Mathematical and Computer Modelling, 49,7/8, 1337-1349

13. Fishman G.S., 1996, Monte Carlo, Concepts, Algorithms and Applications, First Ed. SpringerVerlag

14. Ghanem R.G., Spanos P.D., 1991, Stochastic Finite Elements: A Spectral Approach, Revised Ed. Springer Verlag

15. Guerine A., El Hami A., Fakhfakh T., Haddar M., 2015a, A polynomial chaos method to the analysis of the dynamic behavior of spur gear system, Structural Engineering and Mechanics, 53, 819-831

16. Guerine A., El Hami A., Walha L., Fakhfakh T., Haddar M., 2015b, A perturbation approach for the dynamic analysis of one stage gear system with uncertain parameters, Mechanism and Machine Theory, 92, 113-126

17. Isukapalli S.S., Roy A., Georgopoulos P.G., 1998a, Development and application of methods for assessing uncertainty in photochemical air quality problems, Interim Report for U.S.EPA National Exposure Research Laboratory

18. Isukapalli S.S., Roy A., Georgopoulos P.G., 1998b, Stochastic response surface methods (SRSMs) for uncertainty propagation: application to environmental and biological systems, Risk Analysis, 18, 351-363

19. Le Maître O.P., Knio O.M., Najm H.N., Ghanem R.G., 2001, A stochastic projection method for fluid flow Basic formulation, Journal of Computational Physics, 173, 481-511

20. Lindsley N.J., BerAn P.S., 2005, Increased efficiency in the stochastic interrogation of an uncertain nonlinear aeroelastic system, International Forum on Aeroelasticity and Structural Dynamics, Munich, Germany

21. Papadrakakis M., Papadopoulos V., 1999, Parallel solution methods for stochastic finite element analysis using Monte Carlo simulation, Computer Methods in Applied Mechanics and Engineering, 168, 305-320

22. SaAd G., Ghanem R., Masri S., 2007, Robust system identification of strongly nonlinear dynamics using a polynomial chaos based sequential data assimilation technique, Structural Dynamics and Materials Conference, Honolulu, USA 
23. Sandu A., Sandu C., Ahmadian M., 2006a, Modeling multibody dynamic systems with uncertainties. Part I: numerical application, Multibody System Dynamic, 15, 369-391

24. Sandu C., Sandu A., Ahmadian M., 2006b, Modeling multibody dynamic systems with uncertainties. Part II: theoretical and computational aspects, Multibody System Dynamic, 15, 241-262

25. Sarsri D., Azrar L., Jebbouri A., El Hami A., 2011, Component mode synthesis and polynomial chaos expansions for stochastic frequency functions of large linear FE models, Computers and Structures, 89, 3/4, 346-356

26. Sмith A.H.C., Monti A., Ponci F., 2007, Indirect measurements via a polynomial chaos observer, IEEE Transactions on Instrumentation and Measurement, 56, 743-752

27. Walha L., Fakhfakh T., Haddar M., 2009, Nonlinear dynamics of a two-stage gear system with mesh stiffness fluctuation, bearing flexibility and backlash, Mechanism and Machine Theory, 44, 1058-1069

28. Wiener N., 1938, The homogeneous chaos, American Journal of Mathematics, 60, 897-936

29. Williams M.M.R., 2006, Polynomial chaos functions and stochastic differential equations, Annals of Nuclear Energy, 33, 774-785

30. Xiu D., Karniadakis G.E., 2002, Modeling uncertainty in steady state diffusion problems via generalized polynomial chaos, Computer Methods in Applied Mechanics and Engineering, 191, 4927-4948

31. Xiu D., Karniadakis G.E., 2003, Modelling uncertainty in flow simulations via generalized polynomial chaos, Journal of Computational Physics, 187, 137-167 